\title{
EVIDENCIAS DEL USO DE LA MUSICOTERAPIA Y LA MEMORIA DEL ADULTO MAYOR
}

\begin{abstract}
EVIDENCE OF THE USE OF MUSIC THERAPY AND MEMORY OF THE ELDERLY
\end{abstract}

Recibido diciembre 2016 Aceptado marzo 2017

\section{Autores:}

Gutiérrez Carrillo PI

Estudiante de Maestría en Ciencias de Enfermería Universidad de Guanajuato,

Campus Celaya Salvatierra

Cordillera\#388 InfonavitAlarcón, Código Postal:82 132 ,

Mazatlán, Sinaloa. Teléfono: (044) 6699188414, Correo

electrónico: pamela_igc@hotmail.com

Jiménez González MJ

Maestra en Ciencias de Enfermería

Docente Universidad de Guanajuato,

Campus Celaya Salvatierra

Departamento de Enfermería Clínica. Av. Ing. Barros Sierra No. 201 Esq. Av Baja California, Ejido de Santa María del Refugio. Celaya, Guanajuato C.P. 38140.

Galindo Soto JA

Doctor en Psicología

Docente Universidad de Guanajuato,

Campus Celaya Salvatierra

Departamento de Enfermería Clínica. Av. Ing. Barros Sierra No. 201 Esq. Av. Baja California, Ejido de Santa María del Refugio. Celaya, Guanajuato C.P. 38140.

Palabras clave: musicoterapia, memoria, adulto mayor.

Key words: music therapy, memory, elderly. 
RESUMEN

Con el avance del envejecimiento se observa un decline parcial de las funciones cognitivas, aunque no es un hecho generalizado, un porcentaje relativamente alto de personas experimentan cambios cognitivos, principalmente problemas de memoria. En los últimos años, la musicoterapia ha ganado un mayor reconocimiento dentro de las intervenciones no farmacológicas. En el campo de la geriatría la musicoterapia es capaz de modular factores de la cognición implicando diversas funciones cognitivas. El objetivo de la presente revisión es examinar las evidencias del uso de la musicoterapia para el fortalecimiento de la memoria en la población adulta mayor. Como resultado de la presente revisión se encontró que la musicoterapia ha sido utilizada en varias ocasiones como terapéutica complementaria en adultos mayores, sobre todo en aquellos que presentan algún tipo de alteración cognitiva relevante, los efectos reportados sugieren mejoría en la función cognitiva de la memoria. Sin embargo, hace falta evidencia de esta intervención en adultos mayores sanos cognitivamente.

Palabras claves: musicoterapia, memoria, adulto mayor.

\section{ABSTRACT}

As the aging progresses, there is a partial decline in cognitive functions, although it is not a generalized fact, a relatively high percentage of people experience cognitive changes, mainly memory problems. Music therapy as a non-pharmacological intervention has been shown to have gained in recent years an important site within therapies. In the field of Geriatrics music therapy is able to modulate factors of cognition involving various cognitive functions. The objective of the present review is to examine and determine the evidences of the use of music therapy in the memory of the adult population. As a result of the present review, it was verified that music therapy improved the cognitive function of memory, especially in the memory of older adults with some type of pathology. Although it is necessary to apply this intervention in healthy older adults.

Key words: music therapy, memory, elderly. 


\section{INTRODUCCIÓN}

\llcorner a musicoterapia ha estado presente desde tiempos remotos, su uso ha ido ganando cada vez mayor importancia dentro de las intervenciones no farmacológicas. Las intervenciones basadas en la música en el cuidado de la salud, son generalmente consideradas de bajo riesgo y aceptable para los pacientes de diferentes edades desde bebés hasta ancianos? sus costos son bajos y se pueden introducir con relativa facilidad. ${ }^{2}$ Dentro del campo de la geriatría puede usarse, según el grado de autonomía y funcionamiento de las personas mayores. ${ }^{3}$

La musicoterapia es definida por la National Association for Music Therapy (Asociación Nacional de Musicoterapia) de EEUU en el folleto A Career in Music Therapy (una carrera en musicoterapia) editado por ella como "el uso de la música en la consecución de objetivos terapéuticos, como son la restauración, el mantenimiento y el acrecentamiento de la salud tanto física como mental".

La musicoterapia es referida como activa y pasiva, la primera es aquella donde los participantes se involucran activamente en la música, dichos participantes pueden ser animados a participar en la improvisación musical con instrumentos o la voz, con la danza, actividades de movimiento o cantar. ${ }^{5}$ La musicoterapia pasiva es definida como aquella que abarca técnicas que permiten al participante escuchar música en lugar de ser un colaborador activo. La música utilizada puede ser en vivo o grabada y de cualquier género. ${ }^{6}$

El solo hecho de oír una canción, decidir si nos gusta o no y tocar un instrumento musical, requieren de una serie de procesos neuroanatómicos y neurofisiológicos complejos?? La música forma parte de habilidades cognitivas en humanos, éstas habilidades tienen regiones específicas en el cerebro ${ }^{8}$ es procesada en el cerebro mediante redes neuronales que implican áreas de procesamiento auditivo y motor, y su percepción y ejecución involucran a diversas funciones cognitivas. ${ }^{9}$ Cuando la música se introduce en el interior del oído, la información viaja a través del tallo cerebral y el mesencéfalo hasta llegar al córtex auditivo. La información es procesada por el córtex auditivo primario ( $A B 41$ y 42, incluida la parte media del giro temporal superior) y el córtex auditivo secundario $\left(A B\right.$ 22).$^{10}$

La música compromete todo el cerebro siendo el hemisferio izquierdo quien se encarga de razonar el volumen y la letra musical. El hemisferio derecho es el encargado de coordinar la percepción y la memoria musical, es importante destacar que al escuchar música se aumenta el flujo sanguíneo en el lóbulo temporal y occipital derecho, estimulando el área de Broca (encargada del lenguaje) en hemisferio izquierdo. La corteza prefrontal rostromedial es capaz de procesar, recordar los tonos y de aprender estructuras musicales; el lóbulo temporal derecho se encargará del procesamiento básico del sonido y de separar la armonía de otros tonos musicales?

Se ha demostrado que la música ha sido de utilidad en la rehabilitación y estimulación en diferentes patologías y cuadros clínicos ${ }^{9}$ como la epilepsia, en las diferentes fases de la demencia, la ansiedad, agresividad, calidad de vida, violencia, estrés, problemas de lenguaje, afasia, autismo, esquizofrenia, daño cerebral, movilidad, entre otras.

Resultando una excelente herramienta para el desarrollo de habilidad, pues activa procesos cognitivos, afectivas y sensoriomotores transferibles a otras funciones cognitivas.?

En el campo de la geriatría la musicoterapia es una herramienta de estimulación cognitiva es fundamental, al considerar que el proceso de envejecimiento se asocia con el deterioro cognitivo y afecta a diversos dominios como la memoria, el lenguaje, las funciones ejecutivas, la velocidad de procesamiento de la información," la orientación, la percepción, la atención y la conciencia. ${ }^{12}$ Existe un interés creciente en la identificación de intervenciones que pueden mejorar la función cognitiva de las personas mayores entre ellas la musicoterapia. ${ }^{13}$

\section{8} MMN/Why Enf Neurol Vol. 16. No. 1 enero - abril 2017 
La memoria es un proceso cognitivo a través del cual se codifica, se almacena y se recupera una información determinada o un suceso concreto. La cual implica tres procesos:

- la codificación que es la transformación de la estimulación sensorial en diferentes códigos de almacenamiento;

- la consolidación o almacenamiento que es el resultado de la elaboración de la información previamente codificada con el objetivo de crear un registro temporal o permanente:

- la recuperación que es aquel acceso y evocación verbal o procedimental de la información previamente almacenada. ${ }^{14}$

Con el avance de la edad se observa un descenso parcial de las funciones cognitivas y aunque esto no es un hecho generalizado, existe un porcentaje relativamente alto de personas que experimentan cambios cognitivos, primordialmente problemas de memoria. ${ }^{15}$ El propósito de esta revisión es examinar y determinar las diversas evidencias del uso de la musicoterapia en la memoria de la población adulta mayor.

\section{DESCRIPCIÓN DE LOS ESTUDIOS}

Se revisaron 38 artículos encontrados en la búsqueda de diferentes bases de datos de los cuales 10 artículos fueron analizados para esta revisión (ver cuadro l); de los cuales se obtuvo una muestra total de 501 adultos mayores, con edad media de 69.36 años y 11.44 años de escolaridad media. Los grupos son heterogéneos y muy diversos en cuanto a sus características y tamaño; los participantes en los estudios eran adultos mayores sanos (4 artículos), o con algún tipo de demencia (6 artículos), institucionalizados (2 artículos) e incluso algunos estudios consideraron a los cuidadores de los adultos mayores (1 artículo). ${ }^{16-25}$ Al respecto llama la atención la presencia de demencias en poco más de la mitad de los grupos considerando la edad promedio, otro dato relevante son los niveles de escolaridad, dado que el equivalente a esa cifra representaría un bachillerato inconcluso, situación que dista mucho de nuestro contexto.
Respecto a las características de la intervención se observa (ver cuadro Il) que los estudios consideraron a la memoria como una de sus variables de estudio, sin embargo, esta fue estudiada en sus diferentes dimensiones; y algunos estudios consideraron otras variables como las actividades básicas de la vida diaria (ABVD) o algunas funciones cognitivas. No es de sorprender que el área más trabajada fuera la cognitiva; en cuanto al tipo de musicoterapia la más utilizada fue música activa. Es evidente que las características de la intervención fueron muy variadas en cuanto a la duración de las sesiones, el número de sesiones y la logística de cada sesión, dado que en algunos casos las actividades incluían instrumentos musicales, canto, escuchar música, movimientos rítmicos y los relatos. Todos los estudios mostraron mejoría, sin embargo, algunos de ellos reportaron mejoría en el grupo de comparación o en ambos grupos, lo que dificulta la interpretación de los resultados, dado que no se puede atribuir a la intervención. Para el resto de los casos, la mejoría fue más notoria en los grupos que incluían adultos mayores con algún nivel de demencia y no así para los adultos mayores sanos o al menos en ellos la mejoría no fue evidente.

\section{CONCLUSIONES}

a revisión evidencia los diferentes usos de la musicoterapia durante el envejecimiento, así como los beneficios en la función cognitiva de la memoria sobre todo en la memoria de los adultos mayores que presentan algún tipo de patología. Si bien, los resultados no fueron favorables para los adultos mayores sanos, vale la pena puntualizar que los cambios en ellos pudieron pasar inadvertidos o los instrumentos utilizados no valoraron a profundidad los cambios, por ello los autores sugieren extender esta intervención en grupos de adultos mayores sanos, también recomiendan un mayor rigor científico en el desarrollo de los estudios.

En nuestro contexto, los centros gerontológicos hacen uso de la música y la motricidad de forma empírica, al respecto valdría la pena conformar grupos de trabajo multidisciplinarios para gestionar

Enf Neurol Vol. 16. No. 1 enero - abril 2017 
proyectos de trabajo que puedan ser replicados con el fin de extender los beneficios de la musicoterapia a más adultos mayores.

Se requiere un programa mayormente estructurado creado por profesionales de musicoterapia y respaldado por profesionales de la salud para darle continuidad y evaluar así los pros y contra de dicha intervención.

\section{AGRADECIMIENTOS}

Al Consejo Nacional de Ciencia y Tecnología (CONACYT) por ser un pilar fundamental en la formación de posgrado.

Cuadro I. Análisis comparativo de los grupos de musicoterapia.

\begin{tabular}{|c|c|c|c|c|}
\hline ESTUDIO & Grupo 1 & Grupo 2 & Grupo 3 & Observaciones \\
\hline $\begin{array}{l}\text { Ceccato E } \\
(2012)(16)\end{array}$ & $\begin{array}{l}(n=23) \\
87.2 \text { años }\end{array}$ & $\begin{array}{l}(n=27) \\
85.5 \text { años }\end{array}$ & 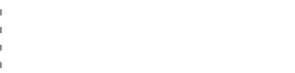 & Ambos grupos con discapacidad. \\
\hline $\begin{array}{l}\text { Chu H. } \\
(2013)(17)\end{array}$ & $(n=52)$ & $(n=52)$ & i & $\begin{array}{l}82 \text { años, } 53 \% \text { mujeres, } 32 \% \text { primaria } \\
\text { Ambos grupos con demencia. }\end{array}$ \\
\hline $\begin{array}{l}\text { Deason RG. } \\
(2012)(18)\end{array}$ & $\begin{array}{l}(\mathrm{n}=14) \\
77.3 \text { años } \\
14 \text { escolaridad } \\
\text { Alzheimer }\end{array}$ & $\begin{array}{l}(n=12) \\
76.3 \text { años } \\
17 \text { escolaridad } \\
\text { Sanos }\end{array}$ & $\begin{array}{l}(n=13) \\
73.7 \text { años } \\
16 \text { escolaridad } \\
\text { Sanos de otro estudio }\end{array}$ & \\
\hline $\begin{array}{l}\text { El Haj M. } \\
(2013)(19)\end{array}$ & $\begin{array}{l}(n=18) \\
75.83 \text { años } \\
5.85 \text { escolaridad } \\
\text { Alzheimer }\end{array}$ & $\begin{array}{l}(n=18) \\
73.61 \text { años } \\
10.56 \text { escolaridad } \\
\text { Sanos }\end{array}$ & ' & \\
\hline $\begin{array}{l}\text { Eldirdiry OS. } \\
(2016)(20)\end{array}$ & $\begin{array}{l}10 \text { parejas de pacientes } \\
\text { y cuidadores. }\end{array}$ & & & $\begin{array}{l}3 \text { parejas de madre-hijo; } 7 \text { parejas de } \\
\text { esposos (5 esposos y } 2 \text { esposas con } \\
\text { demencia). }\end{array}$ \\
\hline $\begin{array}{l}\text { Galdino OTC } \\
(2014)(21)\end{array}$ & $\begin{array}{l}(n=25) \\
76.0 \text { años } \\
4.7 \text { escolaridad } \\
\text { Institucionalizados }\end{array}$ & $\begin{array}{l}(n=17) \\
74.2 \text { años } \\
6.8 \text { escolaridad } \\
\text { No institucionalizados }\end{array}$ & & Ambos grupos cognitivamente sanos. \\
\hline $\begin{array}{l}\text { Hanna-Pladdy B. } \\
\text { (2011) (22) }\end{array}$ & $\begin{array}{l}(n=21) \\
69.7 \text { años } \\
16.2 \text { escolaridad } \\
\text { No músicos }\end{array}$ & $\begin{array}{l}(n=27) \\
69.5 \text { años } \\
17.4 \text { escolaridad } \\
\text { Músicos de baja actividad }\end{array}$ & $\begin{array}{l}(n=22) \\
70.8 \text { años } \\
17.6 \text { escolaridad } \\
\text { Músicos de alta actividad }\end{array}$ & \\
\hline $\begin{array}{l}\text { Särkämö T. } \\
(2013)(23)\end{array}$ & $\begin{array}{l}(n=27) \\
78.5 \text { años } \\
3 \text { escolaridad } \\
\text { Grupo de canto }\end{array}$ & $\begin{array}{l}79.4 \text { años } \\
2.8 \text { escolaridad } \\
\text { Grupo de música de escucha }\end{array}$ & $\begin{array}{l}(n=28) \\
78.4 \text { años } \\
3 \text { escolaridad } \\
\text { Grupo atención habitual }\end{array}$ & $\begin{array}{l}\text { La escolaridad fue medida con } \\
\text { escala de Likert ( } 7 \text { puntos) con } \\
\text { rango: } 1 \text { (educación primaria) } \\
\text { a } 7 \text { (doctorado). }\end{array}$ \\
\hline $\begin{array}{l}\text { Simmons-S NR } \\
(2010)(24)\end{array}$ & $\begin{array}{l}(n=14) \\
77.3 \text { años } \\
14 \text { escolaridad } \\
\text { Alzheimer }\end{array}$ & $\begin{array}{l}(n=13) \\
73.7 \text { años } \\
16 \text { escolaridad } \\
\text { Sanos }\end{array}$ & & $\ldots \ldots$ \\
\hline $\begin{array}{l}\text { Simmons-S NR } \\
(2013)(25)\end{array}$ & $\begin{array}{l}(n=12) \\
81.17 \text { años } \\
14.17 \text { escolaridad } \\
\text { Alzheimer }\end{array}$ & $\begin{array}{l}(n=17) \\
78.63 \text { años } \\
15.5 \text { escolaridad } \\
\text { Sanos }\end{array}$ & & \\
\hline
\end{tabular}


Cuadro II. Análisis comparativo de los programas de musicoterapia en adultos mayores.

\begin{tabular}{|c|c|c|c|c|c|c|}
\hline ESTUDIO & \multicolumn{2}{|c|}{ VARIABLES ESTUDIADAS } & \multicolumn{4}{|c|}{ CARACTERISTICAS DE LA INTERVENCIÓN } \\
\hline Autor & Memoria & Otro & Tipo & Áreas & Duración & Especificaciones \\
\hline $\begin{array}{l}\text { Ceccato E } \\
(2012)(16)\end{array}$ & $\begin{array}{l}\text { Memoria inmediata** } \\
\text { y diferida** }\end{array}$ & $\begin{array}{l}\text { Atención**} \\
\text { ABVD** }^{\star *}\end{array}$ & Música activa & $\begin{array}{l}\text { Cognitiva } \\
\text { Conductual }\end{array}$ & $\begin{array}{l}\text { Sesión: } 45 \text { min } \\
\text { Frecuencia: } 2 / 7 \\
\text { Semanas: } 12\end{array}$ & $\begin{array}{l}\text { Protocolo STAM-Dem: } \\
\text { 1. Asociación estimulo-movimiento, } \\
\text { 2. Reacción a los estímulos acústicos, } \\
\text { 3. Cambiar la atención, } \\
\text { 4. Repetición ordenada e invertida. }\end{array}$ \\
\hline $\begin{array}{l}\text { Chu H. } \\
(2013)(17)\end{array}$ & $\begin{array}{l}\text { Memoria de } \\
\text { corto plazo** }\end{array}$ & $\begin{array}{l}\text { Función cognitiva } \\
\text { global }\end{array}$ & Música activa & $\begin{array}{l}\text { Cognitiva } \\
\text { Conductual }\end{array}$ & $\begin{array}{l}\text { Sesión: } 30 \text { min } \\
\text { Srecuencia: } 2 / 7 \\
\text { Semanas: } 6\end{array}$ & $\begin{array}{l}\text { - Sesión } 1 \text { y 2: Act. con instrumentos musicales. } \\
\text { - Sesión } 3 \text { y } 4 \text { : Act. de canto. } \\
\text { - Sesión } 5 \text { y } 6 \text { : Escuchar música. } \\
\text { - Sesión } 7 \text { y } 8 \text { : Color/sonido. } \\
\text { - Sesión } 9 \text { y } 10: \text { Música/fiesta tradicional. } \\
\text { - Sesión } 11 \text { y 12: Improvisación. }\end{array}$ \\
\hline $\begin{array}{l}\text { Deason RG. } \\
\text { (2012) (18) }\end{array}$ & $\begin{array}{l}\text { Memoria de } \\
\text { reconocimiento** }\end{array}$ & & $\begin{array}{l}\text { Música cantada } \\
\text { y hablada }\end{array}$ & Cognitiva & - Sesión: 30 min & $\begin{array}{l}\text { - } 80 \text { canciones de niños. } \\
\text { y semana de retraso entre la fase de estudio } \\
\text { y de ensayo. }\end{array}$ \\
\hline $\begin{array}{l}\text { El Haj M. } \\
(2013)(19)\end{array}$ & $\begin{array}{l}\text { Memoria } \\
\text { autobiográfica** }\end{array}$ & & $\begin{array}{l}\text { Música favorita } \\
\text { de los } \\
\text { participantes }\end{array}$ & Cognitiva & $\vdots$ & $\begin{array}{l}\text { Expuestos a } 2 \text { minutos de silencio después } 5 \\
\text { minutos de un relato. } \\
\text { - Expuestos a } 2 \text { minutos a la música favorita } \\
\text { después } 5 \text { minutos de un relato. }\end{array}$ \\
\hline $\begin{array}{l}\text { Eldirdiry OS. } \\
(2016)(20)\end{array}$ & Memoria* & & & Cognitiva & : & $\begin{array}{l}\text { - 'Singing for the Brain': Combina aspectos de } \\
\text { la terapia de reminiscencia y la música. } \\
\text { - Canciones familiares que siguen un tema } \\
\text { diferente cada semana. }\end{array}$ \\
\hline $\begin{array}{l}\text { Galdino OIC } \\
\text { (2014)(21) }\end{array}$ & Memoria* & Lenguaje & & Cognitiva & $\begin{array}{l}\text { - Sesión: } 60 \text { min } \\
\text { Frecuencia: } 2 / 7 \\
\text { Semanas: } 24\end{array}$ & - Actividades multisensoriales y cognitivas. \\
\hline $\begin{array}{l}\text { Hanna-Pladdy B. } \\
\text { (2011) (22) }\end{array}$ & $\begin{array}{l}\text { Memoria no verbal* } \\
\text { Memoria de trabajo* }\end{array}$ & $\begin{array}{l}\text { Procesos } \\
\text { ejecutivos } \\
\text { y atención }\end{array}$ & & Cognitiva & $\ldots \ldots$ & $\begin{array}{ll}-1 & -\cdots \\
\vdots \\
\vdots \\
\vdots\end{array}$ \\
\hline $\begin{array}{l}\text { Särkämö T. } \\
(2013)(23)\end{array}$ & $\begin{array}{l}\text { Memoria de corto plazo* } \\
\text { Memoria de trabajo* }\end{array}$ & $\begin{array}{l}\text { Capacidades } \\
\text { cognitivas }\end{array}$ & & Cognitiva & $\begin{array}{l}\text { Sesión: } 90 \text { min } \\
\text { Frecuencia: } 1 / 7 \\
\text { Semanas: } 10 \\
\text { Canciones por } \\
\text { sesión: } 6-10 \\
\text { Número de } \\
\text { participantes: } 10\end{array}$ & $\begin{array}{l}\text { - Grupo de canto: canto, escuchar canciones } \\
\text { conocidas, ejercicios vocales y movimientos } \\
\text { rítmicos. } \\
\text { - Grupo de escucha: reminiscencia y } \\
\text { discusiones. }\end{array}$ \\
\hline $\begin{array}{l}\text { Simmons-S NR } \\
(2010)(24)\end{array}$ & $\begin{array}{l}\text { Memoria de } \\
\text { reconocimiento** }\end{array}$ & & $\begin{array}{l}\text { Música cantada } \\
\text { y hablada }\end{array}$ & Cognitiva & - Sesión: 30 min & $\begin{array}{l}\text { - } 80 \text { canciones infantiles. } \\
\text { Canciones presentadas visualmente, } \\
\text { cantadas y habladas. }\end{array}$ \\
\hline $\begin{array}{l}\text { Simmons-S NR } \\
(2013)(25)\end{array}$ & $\begin{array}{l}\text { Memoria de } \\
\text { reconocimiento** }\end{array}$ & & $\begin{array}{l}\text { Música cantada } \\
\text { y hablada }\end{array}$ & Cognitiva & & $\begin{array}{l}\text { - Canciones infantiles } \\
\text { - Canciones relacionadas a las AIVD. }\end{array}$ \\
\hline
\end{tabular}

$A B V D=$ Actividades básicas de la vida diaria, $A N D=$ Actividades instrumentales de la vida diaria

** Mejoría reportada en el grupo experimental; * Mejoría reportada en el grupo de comparación o en ambos grupos

Fuente: Artículos originales 


\section{REFERENCIAS BIBLIOGRÁFICAS}

1. Robb SL, Burns DS, Carpenter JS. Reporting Guidelines for Music-based Interventions. J Health Psychol (Lon). 2017:16(2): 342-352

2. Matthews S. Dementia and the power of music therapy. Bioethics (USA) 2015: 29(8): 573-579

3. Gómez-Romero M, Jiménez-Palomares M, Rodríguez-Mansilla J, FloresNieto A, Garrido-Ardila E.M. González-LopezArza M.V. Beneficios de la musicoterapia en las alteraciones conductuales de la demencia. Revisión sistemática. Neurología (Esp). 2014;30(20): 1-11.

4. Aguilar L, Barroeta G, Castellanos M, Colmenares D, Hernández N Escritura emocional y musicoterapia pasiva: efectos sobre el estrés de las enfermeras psiquiátricas. Revista científica electrónica de psicología (Ven) 2012;14: $226-50$

5. Vink AC, Bruinsma MS, Scholten R.JPM. Music therapy for people with dementia. Cochrane Libr (Eng). 2013:(3): 1-47.

6. Blackburn R, Bradshaw T. Music therapy for service users with dementia: a critical review of the literature. J Psychiatr Ment HIt (United Kingdom). 2014:21(10): 879-88

7. Montero C. Neuroanatomía musical. Rev Méd Cos Cen (CS). 2010; LXVII(595):493-5

8. Buentello García RM, Martínez Rosas AR, Alonso Vanegas MA. Música y neurociencias. Arch Neurocien (Mex). 2010;15(3): 160-167.

9. Soria-Urios G, Duque P, García-Moreno JM. Música y cerebro (II): evidencias cerebrales del entrenamiento musical. Rev Neurol (Esp). 2011;53:739-746.

10. Soria-Urios G, Duque P. García-Moreno JM. Música y cerebro fundamentos neurocientíficos y trastornos musicales. Rev Neurol (Esp). $2017: 52: 45-55$

11. Galdino TC, Cabral F, Dias M, Wanderley PD, Bento-Torres NVO, PicançoDiniz CW. Beneficial effects of multisensory and cognitive stimulation on age-related cognitive decline in long-term-care institutitos. Clin Interv Aging (New). 2014; 9: 309-327

12. Hui-Chi RN, Hsiu-Hung RN, Fan-Hao RN, Kuei-Min RN. The Effect of Musid Therapy on Cognitive Functioning Among Older Adults: A Systematic Review and Meta-Analysis. J Am Med Dir Assoc (USA). 2015:16(1): 71-77.

13. Hanna-Pladdy B, Mackay A. The Relation Between Instrumental Musical Activity and Cognitive Aging. Neuropsychology (USA). 2011;25(3): 378-386
14. Blázquez JL, Galpasoro N, González B, Lubrini G, Periáñez JA, Ríos M Sánchez I. Tirapu J, Zulaica A. Estimulación cognitiva y rehabilitación neuropsicológica. Barcelona: Editorial UOC; 2009.

15. Pedraza C. Neuropsicología del envejecimiento y las demencias Escritos de Psicología (Esp). $2013: 6(3): 1-4$.

16. Ceccato E, Vigato G, Bonetto C, Bevilacqua A, Pizziolo P, Crociani S, et al. STAM protocol in dementia: a multicenter, single-blind, randomized, and controlled trial. Am J Alzheimers Dis Other Demen (USA). 2012;27(5): 301-10.

17. Chu H, Yang CY, Lin Y, Ou KL, Lee TY, O'Brien AP, et al. The Impact of Group Music Therapy on Depression and Cognition in Elderly Persons With Dementia: A Randomized Controlled Study. Biol Res Nurs (Tai). 2013;16(2): 209-17.

18. Deason RG, Simmons-Stern NR, Frustace BS, Ally BA, Budson AE. Music as a Memory Enhancer: Differences Between Healthy Older Adults and Patients With Alzheimer's Disease. Psychomusicology (USA). 2012;22(2): 175-9.

19. El Haj M, Clément S, Fasotti L, Allain P. Effects of music on autobiographica verbal narration in Alzheimer's disease. J Neurolinguistics (Fra). 2013;26(6) $697-700$.

20. Eldirdiry Osman S, Tischler V, Schneider J. 'Singing for the Brain': A qualitative study exploring the health and well-being benefits of singing for people with dementia and their carers. Dementia (Lon). 2016 Nov:15(6): 1326-1339.

21. Galdino De Oliveira TC, Cabral Soares F, Dias E Dias De Macedo L. Wanderley Picanço Diniz DL, Bento-Torres NVO, Picanço-Diniz CV. Beneficia effects of multisensory and cognitive stimulation on age-related cognitive decline in long-term-care institutions. Clin Interv Aging (Bra). 2014;9: 309-321.

22. Hanna-Pladdy B, Mackay A. The Relation Between Instrumental Musical Activity and Cognitive Aging. Neuropsychology (USA). 2011;25(3): 378-386.

23. Särkämö T, Tervaniemi M, Laitinen $S$, Numminen A, Kurki M, Johnson JK et al. Cognitive, emotional, and social benefits of regular musical activities in early dementia: randomized controlled study. The Gerontologist (Fin). 2013:54(4): 634-650.

24. Simmons-Stern NR, Budson AE, Ally BA. Music as a memory enhancer in patients with Alzheimer's disease. Neuropsychology (USA). 2010;48(10): 2-10

25. Simmons-Stern NR, Deason RG, Brandler BJ, Frustace BS, O'Connor MK. Ally BA, et al. Music-based memory enhancement in Alzheimer's disease promise and limitations. Neuropsychology (USA). 2013;50(14): 3295-303, 